\title{
A Popov Criterion for Networked Systems ${ }^{2}$
}

\author{
Ulf Jönsson, ${ }^{\mathrm{a}, 1}$ Chung-Yao Kao, ${ }^{\mathrm{b}}$ Hisaya Fujioka ${ }^{\mathrm{c}}$ \\ ${ }^{a}$ Optimization and Systems Theory, Royal Institute of Technology, 10044 \\ Stockholm, Sweden. \\ ${ }^{\mathrm{b}}$ Department of Electrical and Electronic Engineering, University of Melbourne, \\ Parkville 3010, VIC Australia \\ ${ }^{\mathrm{c}}$ Graduate School of Informatics, Kyoto University, Kyoto 606-8501, Japan.
}

\begin{abstract}
We consider robustness analysis of heterogeneous and homogeneous networked systems based on integral quadratic constraints (IQCs). First, we show how the analysis decomposes into lower dimensional problems if the interconnection structure is exploited. This generally leads to a significant reduction of the computational complexity. Secondly, by considering a set of IQCs that characterizes the eigenvalues of the interconnection matrices of symmetrically networked systems, we derive a Popov-like criterion for such systems. In particular, when the nodes of the networked system are single-input-single-output linear time-invariant operators, the criterion can be illustrated using a generalized Popov plot. In such cases, the Popov criterion is also a necessary condition in the sense that if the criterion is violated then a destabilizing network with the specified eigenvalue distribution can be constructed.
\end{abstract}

Key words: Networked Control Systems, Robustness, Stability, Integral Quadratic Constraint, Uncertainty, Popov Criteria.

\section{INTRODUCTION}

Many analysis techniques for large scale networked systems are decentralized/decoupled in the sense that important system properties such as stability

$\overline{1}$ Corresponding author. Tel: +46-8-7908450. Fax: +46-8-225320. E-mail address: ulfj@math.kth.se

2 This research was supported by a grant from the Swedish Research Council (VR), the European Commission research project FP6-IST-511368 Hybrid Control (HYCON), and the Australian Research Council. 
can be verified by using criteria which are built on characterization of the individual components such as passivity, gain, or energy dissipation. See e.g. [12]. In this paper, we consider networked systems where network structure can be explicitly exploited to reduce computational complexity and to reveal inherent structure in the problem. The instrument which allows us to carry out the analysis is the so-called integral quadratic constraints (IQCs) [11].

The network structures and decomposition techniques we consider here are similar to previous works on synchronization problems [15, 13], consensus problems [14], vehicle formations [5], Internet congestion control [4, 9, 10], and other network problems with symmetry such as $[1,6]$. One characteristic for all these applications is that it has been possible to derive analysis and design results that are completely decentralized in the sense that conditions for stability and performance can be obtained using only local information and possibly some spectral characteristic of the interconnection matrix. This is a very useful property in the analysis of large scale systems.

Our first contribution is to derive some conditions under which IQC analysis of large scale systems decomposes into analysis conditions involving only the individual local dynamics. In a heterogeneous network, where the local dynamics are different but characterized by the same IQC, we show that the analysis problem decomposes when the interconnection matrix is normal. In an homogeneous network, where we require the nodes to have identical linear dynamics that satisfies a class of IQCs, it turns out that decomposition takes place regardless of the structure of the interconnection matrix. In both cases the decomposition results in great reduction of the computational complexity of the analysis.

The second contribution is to identify a class of Popov criteria for networks with symmetric interconnection matrices. The criterion is obtained by using an IQC that appears in real- $\mu$ analysis $[3,16]$. This IQC is used to characterize that the symmetric interconnection matrix has real eigenvalues within a certain range. For single-input-single-output (SISO) linear time-invariant systems (LTI) the new criterion can be illustrated in a generalized Popov plot with a frequency dependent separating hyperplane. In this case the Popov criterion is a necessary and sufficient characterization of robust network stability in the sense that, if the dynamics of the nodes violates the Popov criterion then there exists a symmetric interconnection matrix with eigenvalue distribution within the sector bound which leads to an unstable networked system.

A related result was obtained in a recent paper. In [8], a separating hyperplane result for stability of heterogeneous LTI systems is shown to correspond to an integral quadratic constraint, which is a special case of our Popov IQC. This IQC was then used to derive, possibly the first, general decomposition result for robust stability analysis of heterogeneous networks. 


\section{Notation and Preliminaries}

The systems considered in the paper are defined on a Hilbert space $\mathcal{H}$ or an extended version of it. Some examples that are common in applications are $\mathbf{L}_{2}^{m}(-\infty, \infty), \mathbf{L}_{2}^{m}[0, \infty)$ and $l_{2}^{m}\left(Z_{+}\right)$. The spatial dimension $m$ is almost always suppressed from the notation.

The inner product of a Hilbert space $\mathcal{H}$ is denoted by $\langle v, w\rangle_{\mathcal{H}}$ and the corresponding norm is $\|v\|_{\mathcal{H}}=\langle v, v\rangle_{\mathcal{H}}^{1 / 2}$. The adjoint of a bounded linear operator $\Phi: \mathcal{H} \rightarrow \mathcal{H}$, denoted as $\Phi^{*}$, is defined by the relation $\langle\Phi v, w\rangle_{\mathcal{H}}=\left\langle v, \Phi^{*} w\right\rangle_{\mathcal{H}}$, for all $v, w \in \mathcal{H}$. A bounded linear operator $\Phi: \mathcal{H} \rightarrow \mathcal{H}$ is called self-adjoint if $\Phi=\Phi^{*}$. A self-adjoint operator $\Pi$ is positive semi-definite if and only if (iff) $\langle v, \Phi v\rangle_{\mathcal{H}} \geq 0$, for all $v \in \mathcal{H}$. It is negative semi-definite iff $-\Phi$ is positive semi-definite. Likewise, a self-adjoint operator $\Phi$ is (strictly) positive definite iff there exists $\epsilon>0$ such that $\langle v, \Phi v\rangle_{\mathcal{H}} \geq \epsilon\|v\|_{\mathcal{H}}^{2}$, for all $v \in \mathcal{H}$. It is negative definite iff $-\Phi$ is positive definite. We use the notation $\Phi \geq 0(\Phi \leq 0$, $\Phi>0, \Phi<0$, respectively) to denote positive semi-definiteness (negative semi-definiteness, positive definiteness, negative definiteness, respectively).

The systems considered in this manuscript will generally have a dynamics given by a bounded linear operator $G: \mathcal{H} \rightarrow \mathcal{H}$ in feedback interconnection with a (possibly nonlinear) bounded operator $\Delta: \mathcal{H} \rightarrow \mathcal{H}$. Here boundedness means that the induced gain

$$
\|\Delta\|=\sup _{v \in \mathcal{H}} \frac{\|\Delta(v)\|_{\mathcal{H}}}{\|v\|_{\mathcal{H}}}
$$

is finite. An important case is when $\Delta \in \mathbf{R H}_{\infty}^{m \times m}$, the space of real rational transfer functions with no poles in the closed right half plane. Then $\Delta$ : $\mathbf{L}_{2}[0, \infty) \rightarrow \mathbf{L}_{2}[0, \infty)$ is bounded, causal, and linear, with $\|\Delta\|=\|\Delta\|_{\mathbf{H}_{\infty}}:=$ $\sup _{\omega \in[0, \infty]} \sigma_{\max }(\Delta(j \omega))$, where $\sigma_{\max }(\cdot)$ denotes the largest singular value. For more background on the above definitions, readers are referred to $[2,7,17]$.

Definition 1 Let $\Pi: \mathcal{H} \times \mathcal{H} \rightarrow \mathcal{H} \times \mathcal{H}$ be a self-adjoint bounded linear operator. We say that $\Delta$ satisfies the IQC defined by $\Pi$ (notation: $\Delta \in \operatorname{IQC}(\Pi)$ ) if and only if

$$
\left\langle\left[\begin{array}{c}
v \\
\Delta(v)
\end{array}\right], \Pi\left[\begin{array}{c}
v \\
\Delta(v)
\end{array}\right]\right\rangle_{\mathcal{H} \times \mathcal{H}} \geq 0, \quad \forall v \in \mathcal{H}
$$

In the same way, we say that $\Delta$ satisfies the strict IQC defined by $\Pi$ (notation: $\Delta \in \mathrm{SIQC}(\Pi))$ if and only if there exists $\epsilon>0$ such that

$$
\left\langle\left[\begin{array}{c}
v \\
\Delta(v)
\end{array}\right], \Pi\left[\begin{array}{c}
v \\
\Delta(v)
\end{array}\right]\right\rangle_{\mathcal{H} \times \mathcal{H}} \geq \epsilon\|v\|_{\mathcal{H}}^{2}
$$


for all $v \in \mathcal{H}$.

The matrix $\Pi$ is often block partitioned into the form

$$
\left[\begin{array}{ll}
\Pi_{11} & \Pi_{12} \\
\Pi_{12}^{*} & \Pi_{22}
\end{array}\right]
$$

where the dimensions of $\Pi_{i j}$ are consistent with those of $v$ and $\Delta(v)$. Furthermore, for a given $\Pi$ with the above partition, we often require the following accompanying operator

$$
\Pi^{s}:=\left[\begin{array}{ll}
0 & I \\
I & 0
\end{array}\right]\left[\begin{array}{ll}
\Pi_{11} & \Pi_{12} \\
\Pi_{12}^{*} & \Pi_{22}
\end{array}\right]\left[\begin{array}{ll}
0 & I \\
I & 0
\end{array}\right]=\left[\begin{array}{ll}
\Pi_{22} & \Pi_{12}^{*} \\
\Pi_{12} & \Pi_{11}
\end{array}\right] .
$$

Definition 2 A feedback interconnection of $G$ and $\Delta$ is said to be robust if $\Delta \in \mathrm{IQC}(\Pi)$ and $G \in \mathrm{SIQC}\left(-\Pi^{s}\right)$. If $G$ is a linear operator, this strict IQC condition on $G$ can equivalently be written: there exists $\epsilon>0$ such that

$$
\left[\begin{array}{c}
G \\
I
\end{array}\right]^{*} \Pi\left[\begin{array}{l}
G \\
I
\end{array}\right] \leq-\epsilon I
$$

The robustness condition generally implies a zero exclusion property which under suitable technical conditions can be used to prove various types of robustness. The most important case is the robust stability criterion in [11].

Theorem 1 ([11]) Consider the feedback interconnection

$$
\begin{aligned}
v & =G w+e \\
w & =\Delta(v)
\end{aligned}
$$

where $G \in \mathbf{R H}_{\infty}^{m \times m}, \Delta: \mathbf{L}_{2 e}^{m}[0, \infty) \rightarrow \mathbf{L}_{2 e}^{m}[0, \infty)$ is a bounded causal operator, and $e \in \mathbf{L}_{2 e}^{m}[0, \infty)$. The interconnection is assumed to be well-posed as defined in [11]. Then the system is input-output stable if there exists a bounded measurable function $\Pi=\Pi^{*}: j \mathbf{R} \rightarrow \mathbf{C}^{2 m \times 2 m}$ such that

(i) $\Delta \in \mathrm{IQC}(\Pi)$,

(ii) $G \in \operatorname{SIQC}\left(-\Pi^{s}\right)$,

(iii) $\Pi_{11} \geq 0$ and $\Pi_{22} \leq 0$.

The Kronecker product is denoted by $\otimes$. Given $\Delta_{k}: \mathcal{H}_{k} \rightarrow \mathcal{H}_{k}, k=1, \cdots, n$, the operator direct sum of $\Delta_{k}$, denoted by $\oplus_{k=1}^{n} \Delta_{k}$, is defined as $\operatorname{diag}\left(\Delta_{1}, \ldots, \Delta_{n}\right)$, 
which should be interpreted as

$$
\left(\oplus_{k=1}^{n} \Delta_{k}\right)(v):=\left[\begin{array}{c}
\Delta_{1}\left(v_{1}\right) \\
\vdots \\
\Delta_{n}\left(v_{n}\right)
\end{array}\right] .
$$

The following simple observation will be useful later.

Lemma 1 Let the Hilbert space $\mathcal{H}$ be a vector space over $\mathbf{R}$ and consider the quadratic form $\sigma(v, w)=\langle v, \Phi w\rangle$, where $\Phi=\Phi^{*}$. The following are equivalent

(i) $\sigma(v, v) \geq 0, \forall v \in \mathcal{H}$

(ii) $\sigma\left(v_{R}+j v_{I}, v_{R}+j v_{I}\right) \geq 0, \forall v_{R}, v_{I} \in \mathcal{H}$

PROOF. The quadratic form satisfies $\sigma(v, w)=\sigma(w, v)$ because $\Phi=\Phi^{*}$ and $\langle v, w\rangle=\langle w, v\rangle .(i) \Rightarrow(i i)$ follows since $\left\langle v_{R}+j v_{I}, \Phi\left(v_{R}+j v_{I}\right)\right\rangle=\left\langle v_{R}, \Phi v_{R}\right\rangle+$ $\left\langle v_{I}, \Pi v_{I}\right\rangle \geq 0$ and both terms on the right hand side are positive. The other direction is obvious.

\section{HETEROGENEOUS NETWORKS}

We consider the system

$$
\begin{aligned}
& u=\Gamma y,
\end{aligned}
$$

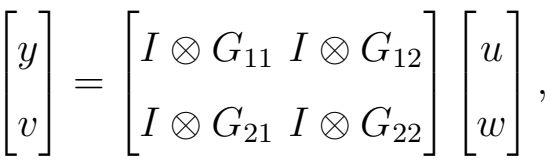

$$
\begin{aligned}
& w=\Delta(v)
\end{aligned}
$$

where $\Delta=\oplus_{k=1}^{n} \Delta_{k}$, and each $\Delta_{k}$ is assumed to satisfy an IQC defined by $\Pi$ $\left(\Delta_{k} \in \mathrm{IQC}(\Pi), k=1, \ldots, n\right)$. The interconnection is modeled as $\Gamma=\hat{\Gamma} \otimes I$, where $\hat{\Gamma} \in \mathbf{R}^{n \times n}$. It is assumed that the spatial dimensions of all operators are consistent. The system (1) represents a network with nodes

$$
G\left(\Delta_{k}\right)=G_{11}+G_{12} \Delta_{k}\left(I-G_{22} \Delta_{k}\right)^{-1} G_{21},
$$

$k=1, \ldots, n$ and the interconnection topology is determined by the matrix $\Gamma$. See Figure 1 for an illustration. The graph description of the system is equivalent to the block diagram description in the lower part of the figure, where $G_{\Gamma}=\hat{\Gamma} \otimes G_{21}\left(I \otimes I-\hat{\Gamma} \otimes G_{11}\right)^{-1} I \otimes G_{12}+I \otimes G_{22}$ is the upper linear fractional transformation within the dashed block. Here it is assumed that $G_{\Gamma}$ is well posed, i.e. $I \otimes I-\hat{\Gamma} \otimes G_{11}$ has a bounded inverse. It can be readily 

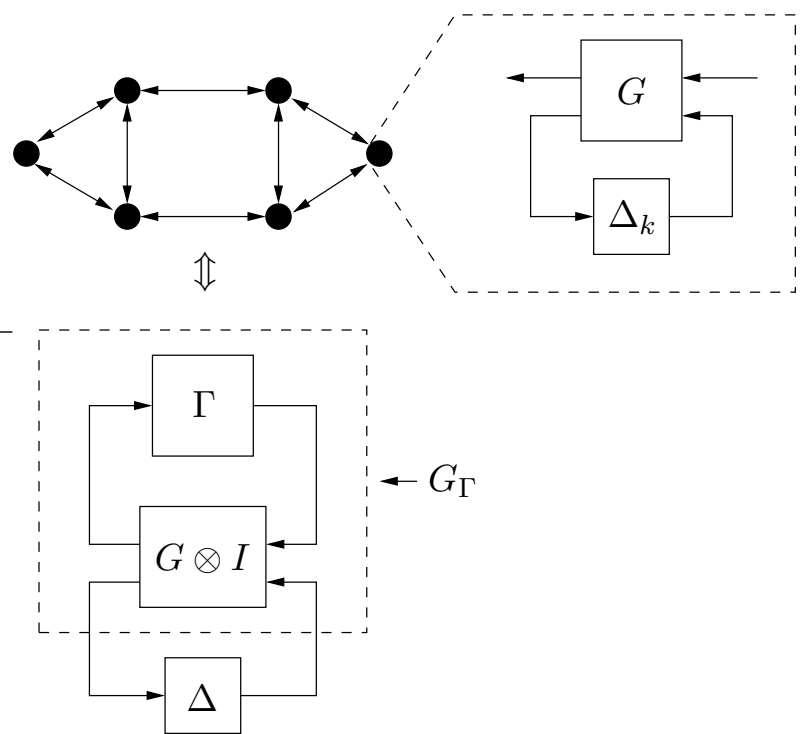

Fig. 1. The networked system and its corresponding block diagram description. verified that $\Delta$ satisfies an IQC defined by

$$
\Pi_{\mathrm{tot}}:=\left[\begin{array}{lll}
I \otimes \Pi_{11} & I \otimes \Pi_{12} \\
I \otimes \Pi_{12}^{*} & I \otimes \Pi_{22}
\end{array}\right] .
$$

Hence, the interconnected system (1) is robust if

$$
G_{\Gamma} \in \operatorname{SIQC}\left(-\Pi_{\text {tot }}^{s}\right) .
$$

Proposition 1 Suppose $\Gamma=\hat{\Gamma} \otimes I$, where $\hat{\Gamma} \in \mathbf{R}^{n \times n}$ satisfies $\hat{\Gamma} \hat{\Gamma}^{T}=\hat{\Gamma}^{T} \hat{\Gamma}$. Then the robustness condition (2) holds if and only if

$$
\left[\begin{array}{c}
G_{\lambda} \\
I
\end{array}\right]^{*} \Pi\left[\begin{array}{c}
G_{\lambda} \\
I
\end{array}\right] \leq-\epsilon I, \quad \forall \lambda \in \operatorname{eig}(\hat{\Gamma})
$$

where $G_{\lambda}=\lambda G_{21}\left(I-\lambda G_{11}\right)^{-1} G_{12}+G_{22}$ and $\operatorname{eig}(\hat{\Gamma})$ denotes the set of eigenvalues of $\hat{\Gamma}$.

Remark 1 The $G_{\lambda}$ are well-posed, i.e. $\left(I-\lambda G_{11}\right)$ has a bounded inverse for $\lambda \in \operatorname{eig}(\hat{\Gamma})$, by the assumption that $G_{\Gamma}$ is well posed. This follows since $G_{\Gamma}=(\Psi \otimes I) G_{\Lambda}\left(\Psi^{*} \otimes I\right)$, where $\Psi$ is a unitary matrix consisting of the eigenvectors of $\hat{\Gamma}$, and $G_{\Lambda}$ is the block-diagonal operator

$$
G_{\Lambda}=\left(\Lambda \otimes G_{21}\right)\left(I \otimes I-\Lambda \otimes G_{11}\right)^{-1}\left(I \otimes G_{12}\right)+I \otimes G_{22} .
$$

PROOF. Since the interconnection matrix is normal, i.e. $\hat{\Gamma} \hat{\Gamma}^{T}=\hat{\Gamma} \hat{\Gamma}^{T}$, we have an eigenvalue decomposition of the form $\hat{\Gamma}=\Psi \Lambda \Psi^{*}$ where $\Psi=\left[\psi_{1} \ldots \psi_{n}\right]$ 
is an $n$-dimensional complex-valued unitary matrix, and $\Lambda$ is a diagonal matrix where the entries of $\Lambda$ are the eigenvalues $\lambda_{k}$ of matrix $\hat{\Gamma}$. We consider the case where $\mathcal{H}$ is a vector space over $\mathbf{R}$. The case where the scalar field is the complex numbers is treated similarly.

Given an arbitrary $w \in \mathcal{H}^{n} \oplus j \mathcal{H}^{n}, w$ can be represented as $\sum_{i=1}^{2} \sum_{k=1}^{n} \alpha_{i, k} \psi_{k} \otimes$ $w_{i, k}$, where $\alpha_{1, k}=1, \alpha_{2, k}=j$, and $w_{i, k} \in \mathcal{H}$. Therefore,

$$
G_{\Gamma} w=(\Psi \otimes I) G_{\Lambda}\left(\Psi^{*} \otimes I\right) w=(\Psi \otimes I) G_{\Lambda} \sum_{i=1}^{2} \sum_{k=1}^{n} \alpha_{i, k} e_{k} \otimes w_{i, k}
$$

where $e_{k}$ is the $k^{t h}$ unit vector and $G_{\Lambda}$ is defined as in (4). Notice that

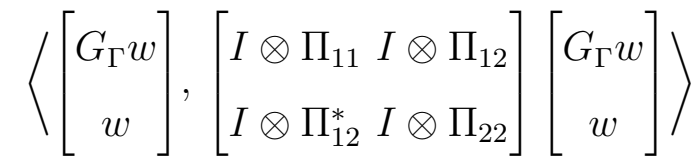

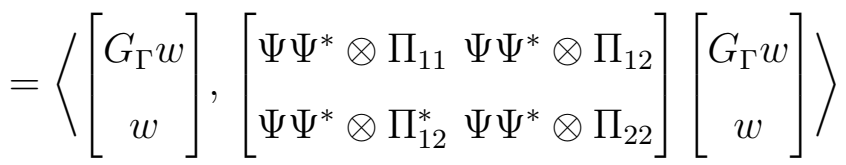

which in turn is equal to

$$
\begin{aligned}
& \left\langle\sum_{i=1}^{2} \sum_{k=1}^{n} \alpha_{i, k} e_{k} \otimes w_{i, k},\left[\begin{array}{c}
G_{\Lambda} \\
I
\end{array}\right]^{*}\left[\begin{array}{c}
I \otimes \Pi_{11} I \otimes \Pi_{12} \\
I \otimes \Pi_{12}^{*} I \otimes \Pi_{22}
\end{array}\right]\left[\begin{array}{c}
G_{\Lambda} \\
I
\end{array}\right] \sum_{i=1}^{2} \sum_{k=1}^{n} \alpha_{k, i} e_{k} \otimes w_{k, i}\right\rangle \\
= & \sum_{i=1}^{2} \sum_{k=1}^{n}\left\langle w_{i, k},\left[\begin{array}{c}
G_{\lambda_{k}} \\
I
\end{array}\right]^{*} \Pi\left[\begin{array}{c}
G_{\lambda_{k}} \\
I
\end{array}\right] w_{i, k}\right\rangle .
\end{aligned}
$$

The $w_{i, k}$ are arbitrary, which by Lemma 1 implies that the quadratic form is negative definite if and only if each term is negative definite. The condition that each term is strictly negative for all $w_{i, k} \in \mathcal{H}$ is equivalent to (3).

As a special case, let us consider the interconnected system

$$
\begin{aligned}
v & =\Gamma w \\
w & =\Delta(v)
\end{aligned}
$$

where $\Delta=\oplus_{k=1}^{n} \Delta_{k}, \Delta_{k} \in \operatorname{IQC}(\Pi)$, and $\Gamma=\hat{\Gamma} \otimes I$.

Corollary 1 Suppose $\Gamma=\hat{\Gamma} \otimes I$, where $\hat{\Gamma} \in \mathbf{R}^{n \times n}$ satisfies $\hat{\Gamma} \hat{\Gamma}^{T}=\hat{\Gamma} \hat{\Gamma}^{T}$. Then the robustness condition

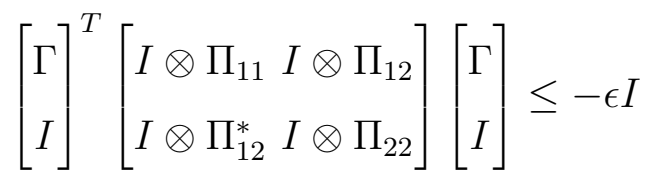


holds if and only if

$$
\left[\begin{array}{c}
\lambda I \\
I
\end{array}\right]^{*} \Pi\left[\begin{array}{c}
\lambda I \\
I
\end{array}\right] \leq-\epsilon I, \quad \forall \lambda \in \operatorname{eig}(\hat{\Gamma})
$$

PROOF. Let $G=\left[\begin{array}{ll}0 & I \\ I & 0\end{array}\right]$. Condition (6) follows immediately by applying $G$ to condition (3).

We can also turn the arguments around and obtain a network Popov criterion for symmetric networked systems.

Corollary 2 (Heterogeneous network Popov criterion) Consider the system (5) with $\Delta=\oplus_{k=1}^{n} \Delta_{k}$ where $\Delta_{k}$ is a bounded causal operator on $\mathbf{L}_{2 e}[0, \infty)$. Suppose there exist bounded measurable matrix functions $X$ and $Y$ defined on $j \mathbf{R}$, where $X(j \omega)=X(j \omega)^{*} \geq \epsilon_{0} I$ for some $\epsilon_{0}>0$ and $Y(j \omega)^{*}=-Y(j \omega)$, such that $\Delta_{k} \in \mathrm{IQC}(\Pi), k=1, \ldots, n$, and $\Pi$ has the following form

$$
\Pi=\left[\begin{array}{cc}
2 X(j \omega) & -(\alpha+\beta) X(j \omega)+Y(j \omega)^{*} \\
-(\alpha+\beta) X(j \omega)+Y(j \omega) & 2 \alpha \beta X(j \omega)
\end{array}\right]
$$

Then the networked system is stable for any symmetric interconnection matrix with eigenvalues satisfying $\operatorname{eig}(\hat{\Gamma}) \in(\alpha, \beta)$, where $\alpha<0 \leq \beta$.

Remark 2 An alternative formulation would be to let $\operatorname{eig}(\hat{\Gamma}) \in[\alpha, \beta]$ and $\Delta_{k} \in \mathrm{SIQC}(\Pi)$.

PROOF. The result follows from Theorem 1 and Corollary 1. Indeed, by the assumptions on $X, Y$, and the spectrum of $\hat{\Gamma}$, we have the equivalent frequency domain condition

$$
\begin{aligned}
{\left[\begin{array}{c}
\lambda I \\
I
\end{array}\right]^{*} \Pi(j \omega)\left[\begin{array}{c}
\lambda I \\
I
\end{array}\right] } & =-2 X(j \omega)(\beta-\lambda)(\lambda-\alpha)-\lambda\left(Y(j \omega)+Y(j \omega)^{*}\right) \\
& =-2 X(j \omega)(\beta-\lambda)(\lambda-\alpha) \leq-\epsilon I, \quad \forall \omega \in[0, \infty]
\end{aligned}
$$

where $\epsilon=\epsilon_{0} \cdot \min _{\lambda \in \operatorname{eig}(\hat{\Gamma})}(\beta-\lambda)(\lambda-\alpha)>0$.

The reason why the result in Corollary 2 is called a Popov criterion is that in the case where each $\Delta_{k}$ is a stable SISO LTI system, the condition can 


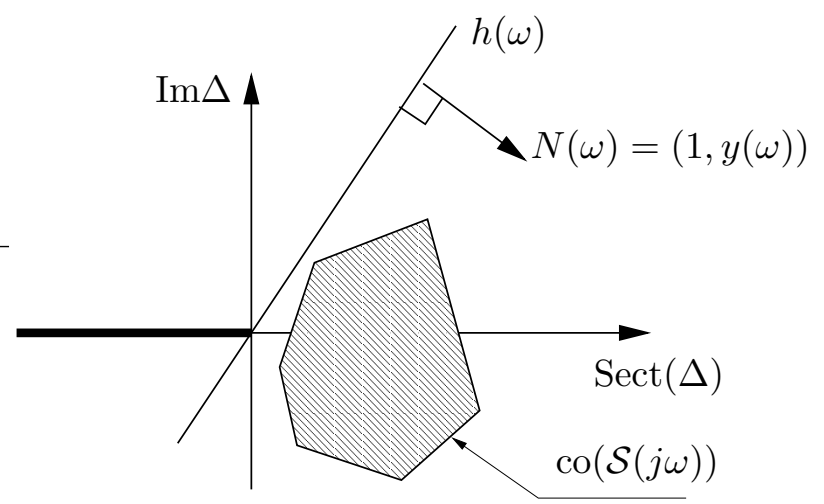

Fig. 2. Illustration of the network Popov condition. The system in (5) with SISO LTI nodes $\Delta_{k}$ is stable if the convex hull of $\mathcal{S}(j \omega)$ can be separated from the negative real axis by a line that goes through the origin.

be verified using a generalized Popov plot. We focus on the case discussed in Remark 2, where $\operatorname{eig}(\Gamma) \in[\alpha, \beta]$, with $\alpha<0 \leq \beta$. In this case the stability condition $\Delta_{k} \in \mathrm{SIQC}(\Pi)$ reduces to: there exists a bounded measurable function $y(\omega) \in \mathbf{R}$ such that $\Delta_{k}$ belongs to

$$
\operatorname{SIQC}\left(\left[\begin{array}{cc}
2 & -(\alpha+\beta)-j y(\omega) \\
-(\alpha+\beta)+j y(\omega) & 2 \alpha \beta
\end{array}\right]\right)
$$

for $k=1, \ldots, n$, which is equivalent to the frequency domain condition

$$
\operatorname{Sect}\left(\Delta_{k}(j \omega)\right)+y(\omega) \operatorname{Im} \Delta_{k}(j \omega)>0, \quad \forall \omega \in[0, \infty]
$$

for $k=1, \ldots, n$, where

$$
\operatorname{Sect}(\Delta)=\alpha \beta|\Delta|^{2}-(\alpha+\beta) \operatorname{Re} \Delta+1
$$

Note that in (8), the same function $y(\omega)$ appears in all $n$ constraints. Namely, all $\Delta_{k}$ must satisfy the same IQC. Figure 2 illustrates the corresponding Popov plot, where $\mathcal{S}(j \omega)$ is a set defined as follows

$$
\mathcal{S}(j \omega)=\left\{\left(\operatorname{Sect}\left(\Delta_{i}(j \omega)\right), \operatorname{Im} \Delta_{i}(j \omega)\right) \mid i=1, \cdots, n\right\}
$$

The interpretation is as follows: the networked system is stable if there exists a frequency varying hyperplane in $\mathbf{R}^{2}$ which separates $\operatorname{co}(\mathcal{S}(j \omega))$ from the negative real axis (here $\operatorname{co}(\cdot)$ denotes the convex hull of a set). It turns out that this condition also provides a necessary condition for robust stability. The next theorem summarizes one of the main results of this paper.

Theorem 2 We have the following two necessary and sufficient characterization of stability of (5) 
(i) Let $\Gamma=\Gamma^{T}$ be given. The networked system (5) is input-output stable for any $\Delta=\oplus_{k=1}^{n} \Delta_{k}$ where each $\Delta_{k} \in \mathbf{R H}_{\infty}^{1 \times 1}$ is a SISO stable LTI system that obeys condition (8) for some bounded measurable function $y(\omega) \in \mathbf{R}$ if and only if all eigenvalues of $\Gamma$ belong to the closed interval $[\alpha, \beta]$, $\alpha<0 \leq \beta$.

(ii) Let $\Delta=\bigoplus_{k=1}^{n} \Delta_{k}$ where $\Delta_{k} \in \mathbf{R} \mathbf{H}_{\infty}^{1 \times 1}$ are given. The networked system is input-output stable for any symmetric interconnection matrix of which all eigenvalues belong to the closed interval $[\alpha, \beta]$, where $\alpha<0 \leq \beta$ if and only if there exists $y(\omega) \in \mathbf{R}$ such that (8) is satisfied.

PROOF. Proof of Theorem 2 is rather lengthy. For the sake of readability, the proof is put in the Appendix.

From the decomposition result, we gain not only insight into how the network structure affects robustness but also a reduction in the computational complexity of the IQC analysis, which perhaps is more important from a practical point of view. The computational saving is obtained at the expense of accuracy; namely, The analysis methodology presented in this section can be conservative because we do not scale the IQCs. To be more specific, suppose we have a convex set $\Pi_{\Delta}$ of IQCs that characterizes each uncertainty, i.e. $\Delta_{k} \in \mathrm{IQC}(\Pi)$, for all $\Pi \in \Pi_{\Delta}$. A more general network robustness condition than (2) can then be formulated as

$$
\left[\begin{array}{c}
G_{\Gamma} \\
I
\end{array}\right]^{*}\left[\begin{array}{ll}
\oplus_{k=1}^{n} \alpha_{k} \Pi_{k,(11)} & \oplus_{k=1}^{n} \alpha_{k} \Pi_{k,(12)} \\
\oplus_{k=1}^{n} \alpha_{k} \Pi_{k,(12)}^{*} & \oplus_{k=1}^{n} \alpha_{k} \Pi_{k,(22)}
\end{array}\right]\left[\begin{array}{c}
G_{\Gamma} \\
I
\end{array}\right] \leq-\epsilon I,
$$

where $\Pi_{k} \in \Pi_{\Delta}$ and $\alpha_{k} \geq 0$. This condition is in general not equivalent to the following decomposed condition: for each $\lambda \in \operatorname{eig}(\hat{\Gamma})$ there exists $\Pi \in \Pi_{\Delta}$ such that

$$
\left[\begin{array}{c}
G_{\lambda} \\
I
\end{array}\right]^{*} \Pi\left[\begin{array}{c}
G_{\lambda} \\
I
\end{array}\right] \leq-\epsilon I
$$

where $G_{\lambda}=\lambda G_{21}\left(I-\lambda G_{11}\right)^{-1} G_{12}+G_{22}$. In the next section we show that this decomposition is possible for general networks if the perturbations are linear and identical.

\section{Homogeneous Networks}

In this section, we consider a case where all perturbations are identical and linear, i.e.,

$$
\Delta=I \otimes \Delta_{0}
$$


where $\Delta_{0}$ is linear. Furthermore, we assume that $\Delta_{0}$ satisfies integral quadratic constraints defined by $\Pi$ 's, where all $\Pi$ belong to a set denoted as $\Pi_{\Delta}$. We have the following result, which is analogous to Proposition 1.

Proposition 2 Consider the networked system (1) with $\Delta$ as in (11) where $\Delta_{0}$ is linear and satisfies $\Delta_{0} \in \mathrm{IQC}(\Pi)$, for all $\Pi \in \Pi_{\Delta}$. Then the network is robust if there exists $\epsilon>0$ such that for each $\lambda \in \operatorname{eig}(\hat{\Gamma})$ there exists $\Pi \in \Pi_{\Delta}$ such that

$$
\left[\begin{array}{c}
G_{\lambda} \\
I
\end{array}\right]^{*} \Pi\left[\begin{array}{c}
G_{\lambda} \\
I
\end{array}\right] \leq-\epsilon I
$$

where $G_{\lambda}=\lambda G_{21}\left(I-\lambda G_{11}\right)^{-1} G_{12}+G_{22}$. This is also a necessary condition if $\Pi_{11} \geq 0$ for all $\Pi \in \Pi_{\Delta}$.

Remark 3 Note that, in contrast to the normality constraint imposed on $\hat{\Gamma}$ in Proposition 1, no restriction is imposed on the network interconnection matrix. That is, the decomposition result holds for any interconnection topology.

PROOF. Consider a Schur decomposition of the coupling matrix $\hat{\Gamma}=\Psi U \Psi^{*}$, where $\Psi=\left[\psi_{1} \ldots \psi_{n}\right]$ is unitary and $U$ is upper triangular. First notice that $\Delta=I \otimes \Delta_{0}$ satisfies the IQC defined by

$$
\Pi_{\text {tot }}=\left[\begin{array}{cc}
\Psi \otimes I & 0 \\
0 & \Psi \otimes I
\end{array}\right]\left[\begin{array}{l}
\oplus_{k=1}^{n} \alpha_{k} \Pi_{k,(11)} \oplus_{k=1}^{n} \alpha_{k} \Pi_{k,(12)} \\
\oplus_{k=1}^{n} \alpha_{k} \Pi_{k,(12)}^{*} \oplus_{k=1}^{n} \alpha_{k} \Pi_{k,(22)}
\end{array}\right]\left[\begin{array}{cc}
\Psi^{*} \otimes I & 0 \\
0 & \Psi^{*} \otimes I
\end{array}\right]
$$

where $\alpha_{k} \geq 0$ and $\Pi_{k} \in \Pi_{\Delta}$. Indeed, let $v=\left[v_{1}^{T}, \cdots, v_{n}^{T}\right]^{T}$, we have

$$
\left\langle\left[\begin{array}{c}
v \\
\Delta(v)
\end{array}\right], \Pi_{\text {tot }}\left[\begin{array}{c}
v \\
\Delta(v)
\end{array}\right]\right\rangle=\sum_{k=1}^{n} \alpha_{k}\left\langle\left[\begin{array}{c}
\check{v}_{k} \\
\Delta_{0} \check{v}_{k}
\end{array}\right], \Pi_{k}\left[\begin{array}{c}
\check{v}_{k} \\
\Delta_{0} \check{v}_{k}
\end{array}\right]\right\rangle \geq 0
$$

where $\check{v}_{k}=\sum_{j=1}^{n} \bar{\psi}_{k, j} v_{j}$ and $\psi_{k, j}$ is the $j^{t h}$ element of $\psi_{k}$. The inequality follows from Lemma 1 .

An arbitrary $w \in \mathcal{H} \oplus j \mathcal{H}$ can be represented as $w=\sum_{i=1}^{2} \sum_{k=1}^{n} \beta_{i, k} \psi_{k} \otimes w_{i, k}$, $\beta_{1, k}=1, \beta_{2, k}=j$, and $w_{i, k} \in \mathcal{H}$. Therefore, we have

$$
G_{\Gamma} w=(\Psi \otimes I) G_{U}\left(\Psi^{*} \otimes I\right) w=(\Psi \otimes I) G_{U} \sum_{i=1}^{2} \sum_{k=1}^{n} \beta_{i, k} e_{k} \otimes w_{i, k}
$$


where $e_{k}$ is the $k^{t h}$ unit vector and $G_{U}=\left(U \otimes G_{21}\right)\left(I \otimes I-U \otimes G_{11}\right)^{-1}(I \otimes$ $\left.G_{12}\right)+I \otimes G_{22}$. It can be shown that

$$
\left\langle\left[\begin{array}{c}
G_{\Gamma} w \\
w
\end{array}\right], \Pi_{\text {tot }}\left[\begin{array}{c}
G_{\Gamma} w \\
w
\end{array}\right]\right\rangle=\langle\bar{w}, \Upsilon \bar{w}\rangle
$$

where $\bar{w}=\sum_{i=1}^{2} \sum_{k=1}^{n} \beta_{i, k} e_{k} \otimes w_{i, k}$ and

$$
\Upsilon=\left[\begin{array}{c}
G_{U} \\
I
\end{array}\right]^{*}\left[\begin{array}{ll}
\oplus_{k=1}^{n} \alpha_{k} \Pi_{k,(11)} & \oplus_{k=1}^{n} \alpha_{k} \Pi_{k,(12)} \\
\oplus_{k=1}^{n} \alpha_{k} \Pi_{k,(12)}^{*} & \oplus_{k=1}^{n} \alpha_{k} \Pi_{k,(22)}
\end{array}\right]\left[\begin{array}{c}
G_{U} \\
I
\end{array}\right]
$$

Hence, by Lemma 1 , it follows that the condition $G_{\Gamma} \in \mathrm{SIQC}\left(-\Pi_{\text {tot }}\right)$ holds if and only if $\Upsilon \leq-\epsilon I$ for some $\epsilon>0$. Note that $G_{U}$ is an upper triangular system, where the diagonal blocks are $G_{\lambda_{k}}, \lambda_{k} \in \operatorname{eig}(\hat{\Gamma})$. Proposition 2 now follows from the next lemma.

Lemma 2 Let

$$
G=\left[\begin{array}{cccc}
G_{11} & G_{12} & \cdots & G_{1 n} \\
0 & G_{22} & \cdots & G_{2 n} \\
\vdots & \vdots & \ddots & \vdots \\
0 & 0 & \cdots & G_{n n}
\end{array}\right]
$$

be an upper triangular system. Consider the following two statements:

$$
\left[\begin{array}{l}
G \\
I
\end{array}\right]^{*}\left[\begin{array}{ll}
\oplus_{k=1}^{n} \alpha_{k} \Pi_{k,(11)} & \oplus_{k=1}^{n} \alpha_{k} \Pi_{k,(12)} \\
\oplus_{k=1}^{n} \alpha_{k} \Pi_{k,(12)}^{*} & \oplus_{k=1}^{n} \alpha_{k} \Pi_{k,(22)}
\end{array}\right]\left[\begin{array}{c}
G \\
I
\end{array}\right] \leq-\epsilon I
$$

(ii) $\left[\begin{array}{c}G_{k k} \\ I\end{array}\right]^{*} \Pi_{k}\left[\begin{array}{c}G_{k k} \\ I\end{array}\right] \leq-\epsilon I, \quad$ for $k=1, \ldots n$.

In general, we have statement (ii) implies statement $(i)$. Moreover, if $\Pi_{k,(11)} \geq$ $0, k=1, \ldots, n$, then the two statements are equivalent.

PROOF. Define $\mathrm{IQC}_{k}$ to be

$$
\mathrm{IQC}_{k}=\left[\begin{array}{c}
G_{k k} \\
I
\end{array}\right]^{*} \Pi_{k}\left[\begin{array}{c}
G_{k k} \\
I
\end{array}\right], \quad k=1, \cdots, n .
$$


Then the $(1,1)$ block of the left hand side of the inequality in $(i)$ becomes $\alpha_{1} \mathrm{IQC}_{1}$ which is negative definite if statement $(i i)$ holds. The upper left block obtained by considering the first two block rows and block columns becomes

$$
\left[\begin{array}{cc}
\alpha_{1} \mathrm{IQC}_{1} & \alpha_{1}\left(G_{11}^{*} \Pi_{1,(11)} G_{12}+\Pi_{1,(12)} G_{12}\right) \\
(\cdot)^{*} & \alpha_{2} \mathrm{IQC}_{2}+\alpha_{1} G_{12}^{*} \Pi_{1,(11)} G_{12}
\end{array}\right]
$$

which can be made negative definite by making $\alpha_{2} / \alpha_{1}$ sufficiently large. The proof of the implication $(i i) \Rightarrow(i)$ follows by continuing this construction until all $n$ block rows and columns are taken into account.

From $(2,2)$ block of $(13)$, we see that if $\Pi_{1,(11)} \geq 0$, then $\mathrm{IQC}_{2}<0$ is required for (13) to be negative definite. In general, it can be shown that the $(\mathrm{k}, \mathrm{k})$ block of the left hand side of the inequality in $(i)$ has the form $\alpha_{k} \mathrm{IQC}_{k}+$ $\sum_{i=1}^{k-1} \alpha_{i} G_{i k}^{*} \Pi_{i,(11)} G_{i k}$. Thus, $(i) \Rightarrow(i i)$ if $\Pi_{k,(11)} \geq 0$.

Finally, we will investigate Popov criteria for homogeneous networks. The following corollary is analogous to Corollary 2.

Corollary 3 (Homogeneous network Popov criterion) Consider the system (5) with $\Delta=I \otimes \Delta_{0}$ as in (11) where $\Delta_{0}$ is a bounded causal linear operator on $\mathbf{L}_{2 e}[0, \infty)$. Suppose there exist bounded measurable matrix functions $X$ and $Y$ defined on $j \mathbf{R}$, where $X(j \omega)=X(j \omega)^{*} \geq \epsilon_{0} I$ for some $\epsilon_{0}>0$ and $Y(j \omega)^{*}=-Y(j \omega)$, such that $\Delta_{0} \in \operatorname{IQC}(\Pi)$, and $\Pi$ is of the form (7). Then the networked system is stable for any interconnection matrix satisfying $\operatorname{eig}(\hat{\Gamma}) \in(\alpha, \beta)$ where $\alpha<0 \leq \beta$.

Remark 4 Alternatively, we can let $\operatorname{eig}(\hat{\Gamma}) \in[\alpha, \beta]$ and $\Delta_{0} \in \operatorname{SIQC}(\Pi)$.

It turns out that the homogeneous network Popov criterion also gives a necessary and sufficient characterization of robust stability if the plant is a SISO LTI plant. This result is completely analogous to Theorem 2 and is omitted. However, the stability characterization will have a simpler graphical description that will be briefly discussed next. As before, we consider the case in Remark 4 , where the eigenvalues of $\hat{\Gamma}$ belong to the close interval $[\alpha, \beta]$ with $\alpha<0 \leq \beta$. In this case, the stability condition $\Delta_{0} \in \mathrm{SIQC}(\Pi)$ reduces to the condition: there exists a bounded measurable function $y(\omega) \in \mathbf{R}$ such that

$$
\operatorname{Sect}\left(\Delta_{0}(j \omega)\right)+y(\omega) \operatorname{Im}\left(\Delta_{0}(j \omega)\right)>0, \quad \forall \omega \in[0, \infty]
$$

where the function $\operatorname{Sect}(\cdot)$ is defined in (9). It follows from the proof of Theorem 2 that this stability condition is satisfied iff the Nyquist curve $\Delta_{0}(j \omega)$ satisfies $\Delta_{0}(j \omega) \notin\left\{s \in \mathbf{C}: \operatorname{Im} s=0 ; \operatorname{Re} s \in\left(-\infty, \alpha^{-1}\right] \cup\left[\beta^{-1}, \infty\right)\right\}$ when $\beta \neq 0$, and $\Delta_{0}(j \omega) \notin\left\{s \in \mathbf{C}: \operatorname{Im} s=0 ; \operatorname{Re} s \in\left(-\infty, \alpha^{-1}\right]\right\}$ when $\beta=0$. 


\section{Concluding Remarks}

We have identified network configurations for which IQC analysis reduces to lower dimensional analysis problems. As an interesting application we showed how this can be used to derive a generalized Popov criterion for networked systems that in some cases are both necessary and sufficient for robust network stability. It is an open issue whether it is possible to obtain similar reductions or approximations thereof for systems with heterogeneous dynamics and arbitrary interconnection topology.

\section{References}

[1] R. Cogill, S. Lall, and P. A. Parrilo. On structured semidefinite programs for the control of symmetric systems. In Proceedings of the Allerton Conference on Communication, Contr ol, and Computing, pages 15361545, 2003.

[2] C.A. Desoer and M. Vidyasagar. Feedback Systems: Input-Output Properties. Academic Press, New York, 1975.

[3] M. K. H. Fan, A. L. Tits, and J. C. Doyle. Robustness in the presence of mixed parametric uncertainty and unmodeled dynamics. IEEE Transactions on Automatic Control, 36(1):25-38, 1991.

[4] X. Fan, M. Arcat, and J. T. Wen. Robustness of network flow control against disturbances and time-delay. Systems and Control Letters, 53:1329, 2004.

[5] J. A. Fax and R. M. Murray. Information flow and cooperative control of vehicle formations. IEEE Transactions on Automatic Control, 49(9):1465 - 1476, 2004.

[6] A. Gattami and R. Murray. A frequency domain condition for stability of interconnected MIMO systems. In Proceedings of the 2004 American Control Conference, pages 3723-3727, Boston, MA, USA, 2004.

[7] E. Kreyszig. Introductory Functional Analysis with Applications. John Wiley \& Sons, New York, 1978.

[8] I. Lestas and G. Vinnicombe. Scalable robustness for consensus protocols with heterogenous dynamics. In Proceedings of the 16th IFAC World Congress, Prague, Czech Republic, 2004.

[9] I. Lestas and G. Vinnicombe. On the scalable stability of nonsymmetric heterogeneous networks. In IEEE Conference on Decision and Control, Seville, Spain, 2005.

[10] S. H Low, F. Paganini, and J. C. Doyle. Internet congestion control. Control Systems Magazine, 22(1):28-43, 2002.

[11] A. Megretski and A. Rantzer. System analysis via integral quadratic constraints. IEEE Transactions on Automatic Control, 42(6):819-830, June 1997. 
[12] P. Moylan and D. Hill. Stability criteria for large-scale systems. IEEE Transactions on Automatic Control, 23(2):143-149, 1978.

[13] A. Pogromsky and H. Nijmeijer. Cooperative oscillatory behavior of mutually coupled dynamical systems. IEEE Transactions on Circuits and Systems-I: Fundamental Theory and Applications, 48(2):152-162, 2001.

[14] R. Olfati Saber and R. M. Murray. Consensus problems in networks of agents with switching topology and time-delays. IEEE Transactions On Automatic Control, 2004.

[15] C. W. Wu and L. O. Chua. Synchronization in an array of linearly coupled dynamical systems. IEEE Transactions on Circuits and Systems-I: Fundamental Theor $y$ and Applications, 42(8):430-447, 1995.

[16] P. M. Young, M. P. Newlin, and J. C. Doyle. Practical computation of the mixed $\mu$ problem. In Proceedings of the American Control Conference, Chicago, Illinois, 1992.

[17] K. Zhou, J.C. Doyle, and K. Glover. Robust and Optimal Control. Prentice Hall, Upper Saddle River, New Jersey, 1996.

\section{Appendix: Proof of Theorem 2}

The sufficiency of both $(i)$ and $(i i)$ follows from Corollary 2 and Remark 2 . The main idea behind the proof of necessity is to derive an equivalent characterization of violation of condition (8). Counter examples can then be constructed to show the necessity.

Criterion (8) is satisfied if and only if (since $y(\omega)$ is any measurable function) $\cap_{\omega \in[0, \infty]} C(\omega) \neq \emptyset$, where

$$
C(\omega)=\left\{x=1: \exists y \in \mathbf{R} \text { s.t. } x \operatorname{Sect}\left(\Delta_{k}(j \omega)\right)+y \operatorname{Im} \Delta_{k}(j \omega)>0 ; k=1, \ldots, n\right\} .
$$

The function Sect $(\cdot)$ is defined in (9). Hence criterion (8) is violated if and only if there exists $\omega_{0} \in[0, \infty]$ such that $C\left(\omega_{0}\right)=\emptyset$. It follows that the criterion is violated if and only if there exists $\omega_{0} \in[0, \infty]$ such that the convex sets

$$
C_{1}:=\left\{x \in \mathbf{R}^{n}: x_{k}=\operatorname{Sect}\left(\Delta_{k}\left(j \omega_{0}\right)\right)+y \operatorname{Im} \Delta_{k}\left(j \omega_{0}\right) ; y \in \mathbf{R}\right\}
$$

and $C_{2}:=\left\{x \in \mathbf{R}^{n}: x_{k}>0\right\}$ are disjoint. Note that $C_{2}$ is open. Hence, by the separating hyperplane theorem an equivalent condition is that there exists a nonzero $z \in \mathbf{R}^{n}$ such that $z_{k} \geq 0$ and $z^{T} x \leq 0$ for all $x \in C_{1}$. In other words,

$$
\sum_{k=1}^{n} z_{k}\left(\operatorname{Sect}\left(\Delta_{k}\left(j \omega_{0}\right)\right)+y \operatorname{Im} \Delta_{k}\left(j \omega_{0}\right)\right) \leq 0
$$

for all $y \in \mathbf{R}$. Hence, the stability criterion is violated if and only if there exists $\omega_{0} \in[0, \infty]$ and $z_{k} \geq 0, k=1, \ldots, n$ of which at least one $z_{k}$ is nonzero 
such that

$$
\sum_{k=1}^{n} z_{k} \operatorname{Im} \Delta_{k}\left(j \omega_{0}\right)=0, \quad \sum_{k=1}^{n} z_{k} \operatorname{Sect}\left(\Delta_{k}\left(j \omega_{0}\right)\right) \leq 0 .
$$

Note that it is no restriction to assume that $\sum_{k=1}^{n} z_{k}=1$.

Necessity for statement $(i)$ : From the above discussion it follows that condition (8) is violated if and only if there exists $\omega_{0}$ such that $\operatorname{co}\left(\mathcal{S}\left(j \omega_{0}\right)\right) \cap \mathbf{R}_{-} \neq \emptyset$, where $\mathbf{R}_{-}=\left\{\left(x_{1}, x_{2}\right) \in \mathbf{R}^{2}: x_{1} \in(-\infty, 0], x_{2}=0\right\}$ and $\mathcal{S}(j \omega)$ was defined in (10). Now, let $\lambda_{0} \notin[\alpha, \beta]$ be a nonzero eigenvalue of $\Gamma$. Let $\Delta_{0} \in \mathbf{R} \mathbf{H}_{\infty}^{1 \times 1}$ satisfy $\operatorname{Im} \Delta_{0}(j \omega) \neq 0$ for all $\omega$ except $\omega=\omega_{0}$, and $\operatorname{Re} \Delta_{0}\left(j \omega_{0}\right)=\mu \lambda_{0}^{-1}$. Consider $\Delta=I \otimes \Delta_{0}$. Clearly, for this $\Delta$, the set $\operatorname{co}(\mathcal{S}(j \omega))$ reduces to a point $\left(\operatorname{Sect}\left(\Delta_{0}(j \omega)\right), \operatorname{Im} \Delta_{0}(j \omega)\right)$. Note that $\operatorname{co}(\mathcal{S}(j \omega))$ may intersect $\mathbf{R}_{-}$only at $\omega=\omega_{0}$ since $\operatorname{Im} \Delta(j \omega)=0$ only at $\omega_{0}$. Furthermore, suppose $\beta \neq 0$. Then

$$
\operatorname{Sect}\left(\Delta_{0}\left(j \omega_{0}\right)\right)=\alpha \beta\left(\mu \lambda_{0}^{-1}-\alpha^{-1}\right)\left(\mu \lambda_{0}^{-1}-\beta^{-1}\right) .
$$

Since $\lambda_{0} \notin[\alpha, \beta]$, we have $\lambda_{0}^{-1} \in\left(\alpha^{-1}, \beta^{-1}\right)$; therefore, $\operatorname{Sect}\left(\Delta_{0}\left(j \omega_{0}\right)\right)>0$ if $\mu=1$. In the case where $\beta=0$, we have $\lambda_{0}^{-1} \in\left(\alpha^{-1}, 0\right) \cup(0, \infty)$. Hence, $\operatorname{Sect}\left(\Delta_{0}\left(j \omega_{0}\right)\right):=-\alpha \mu \lambda_{0}^{-1}+1$ is larger than 0 when $\mu=1$. Thus, by construction, $\Delta$ satisfies condition (8) in a neighborhood of $\mu=1$.

In order to prove that the closed loop network in fact corresponds to an unstable system we let $v_{0}$ be the eigenvector corresponding to the eigenvalue $\lambda_{0}$. Then $\Gamma \Delta\left(j \omega_{0}\right) v_{0}=\mu v_{0}$ so the Nyquist locus of $\Gamma \Delta$ crosses the critical point $s=1$ when $\mu=1$ and the system is at best neutrally stable and not input-output stable.

Necessity for statement (ii): Note that (15) holds if and only if the objective value of the following linear program (LP) is non-positive:

$$
\operatorname{minimize} \sum_{k=1}^{n} z_{k} \operatorname{Sect}\left(\Delta_{k}\left(j \omega_{0}\right)\right) \quad \text { subject to }\left\{\begin{array}{l}
\sum_{k=1}^{n} z_{k} \operatorname{Im} \Delta_{k}\left(j \omega_{0}\right)=0 \\
\sum_{k=1}^{n} z_{k}=1 \\
z_{k} \geq 0
\end{array}\right.
$$

This LP is of the standard form and it is well known that there either exists an optimal solution with at most two nonzero $z_{k}$ or the problem is infeasible (i.e., condition (8) is satisfied). The stability condition is thus violated if there exists $\omega_{0} \in[0, \infty]$, a pair $k_{1}, k_{2}$, and $z \in[0,1)$ such that

$$
\begin{aligned}
& z \operatorname{Im} \Delta_{k_{1}}\left(j \omega_{0}\right)+(1-z) \operatorname{Im} \Delta_{k_{2}}\left(j \omega_{0}\right)=0, \\
& z \operatorname{Sect}\left(\Delta_{k_{1}}\left(j \omega_{0}\right)\right)+(1-z) \operatorname{Sect}\left(\Delta_{k_{2}}\left(j \omega_{0}\right)\right) \leq 0 .
\end{aligned}
$$

We can use this to construct examples to show the necessity of $(i i)$. There are two possible cases 
(i) Suppose $z=0$. Without loss of generality, let $k_{2}=1$, i.e., the condition

$$
\operatorname{Im} \Delta_{1}\left(j \omega_{0}\right)=0, \text { and } \operatorname{Sect}\left(\Delta_{1}\left(j \omega_{0}\right)\right) \leq 0
$$

holds. Consider the network with $\Delta=\oplus_{k=1}^{n} \Delta_{k}$ and $\Gamma=\operatorname{diag}(\delta, 0, \ldots, 0)$. The first condition in (17) implies that $\Delta_{1}\left(j \omega_{0}\right) \in \mathbf{R}$. Furthermore, suppose $\beta \neq 0$. Then the second condition in (17) implies that $\Delta_{1}\left(j \omega_{0}\right) \in$ $\left(-\infty, \alpha^{-1}\right] \cup\left[\beta^{-1}, \infty\right)$. If $\beta=0$, then the condition implies $\Delta_{1}\left(j \omega_{0}\right) \leq \alpha^{-1}$. In either case, there exists $\delta \in[\alpha, \beta]$ such that $\delta \Delta_{1}\left(j \omega_{0}\right)=1$. Thus, the system is at best neutrally stable and not input-output stable.

(ii) Suppose that $z$ is nonzero. Without loss of generality, assume $k_{1}=1$ and $k_{2}=2$. Furthermore, we may assume that neither $\operatorname{Im} \Delta_{1}\left(j \omega_{0}\right)$ nor $\operatorname{Im} \Delta_{2}\left(j \omega_{0}\right)$ is equal to 0 . Otherwise, either $\Delta_{1}\left(j \omega_{0}\right)$ or $\Delta_{2}\left(j \omega_{0}\right)$ must satisfy the conditions stated in (17) and a destabilizing network connection matrix $\Gamma$ can be constructed following the arguments in (i).

Let $\Gamma=\operatorname{diag}(\beta I+\tilde{\Gamma}, 0, \ldots, 0)$, where $\tilde{\Gamma}$ is a $2 \times 2$ symmetric matrix whose elements are of the forms

$$
\begin{aligned}
& \tilde{\gamma}_{11}=\mu(\alpha-\beta) \frac{z\left|1-\beta \Delta_{1}\left(j \omega_{0}\right)\right|^{2}}{z\left|1-\beta \Delta_{1}\left(j \omega_{0}\right)\right|^{2}+(1-z)\left|1-\beta \Delta_{2}\left(j \omega_{0}\right)\right|^{2}}, \\
& \tilde{\gamma}_{22}=\mu(\alpha-\beta) \frac{(1-z)\left|1-\beta \Delta_{2}\left(j \omega_{0}\right)\right|^{2}}{z\left|1-\beta \Delta_{1}\left(j \omega_{0}\right)\right|^{2}+(1-z)\left|1-\beta \Delta_{2}\left(j \omega_{0}\right)\right|^{2}}, \\
& \tilde{\gamma}_{12}=\sqrt{\tilde{\gamma}_{11} \tilde{\gamma}_{22}} .
\end{aligned}
$$

Since $\operatorname{Im} \Delta_{1}\left(j \omega_{0}\right)$ and $\operatorname{Im} \Delta_{2}\left(j \omega_{0}\right)$ are not equal to zero, $\tilde{\gamma}_{11}$ and $\tilde{\gamma}_{22}$ are well defined. Also note that the eigenvalues of $\Gamma$ are $\{\beta, \mu \alpha+(1-\mu) \beta\}$, both of which belong to the interval $[\alpha, \beta]$ for $\mu \in[0,1]$. Given matrix $\Gamma$, we have

$$
\begin{aligned}
\operatorname{det}\left(I-\Gamma \Delta\left(j \omega_{0}\right)\right) & =\operatorname{det}\left(I-\beta \Delta\left(j \omega_{0}\right)-\tilde{\Gamma} \Delta\left(j \omega_{0}\right)\right) \\
& =\operatorname{det}\left(I-\tilde{\Gamma} \Delta\left(j \omega_{0}\right)\left(I-\beta \Delta\left(j \omega_{0}\right)\right)^{-1}\right) \operatorname{det}\left(I-\beta \Delta\left(j \omega_{0}\right)\right)
\end{aligned}
$$

Denote $\Delta\left(j \omega_{0}\right)\left(I-\beta \Delta\left(j \omega_{0}\right)\right)^{-1}$ by $\bar{\Delta}\left(j \omega_{0}\right)$, which can be expressed as

$$
\left[\begin{array}{cc}
\frac{\Delta_{1}\left(j \omega_{0}\right)}{1-\beta \Delta_{1}\left(j \omega_{0}\right)} & 0 \\
0 & \frac{\Delta_{2}\left(j \omega_{0}\right)}{1-\beta \Delta_{2}\left(j \omega_{0}\right)}
\end{array}\right]:=\left[\begin{array}{cc}
\bar{\Delta}_{1}\left(j \omega_{0}\right) & 0 \\
0 & \bar{\Delta}_{2}\left(j \omega_{0}\right)
\end{array}\right] \text {. }
$$

The determinant of $I-\tilde{\Gamma} \Delta\left(j \omega_{0}\right)\left(I-\beta \Delta\left(j \omega_{0}\right)\right)^{-1}$ is then equal to

$$
\begin{aligned}
& \operatorname{det}\left(\left[\begin{array}{cc}
1-\tilde{\gamma}_{11} \bar{\Delta}_{1}\left(j \omega_{0}\right) & -\tilde{\gamma}_{12} \bar{\Delta}_{2}\left(j \omega_{0}\right) \\
-\tilde{\gamma}_{12} \bar{\Delta}_{1}\left(j \omega_{0}\right) & 1-\tilde{\gamma}_{22} \bar{\Delta}_{2}\left(j \omega_{0}\right)
\end{array}\right]\right) \\
& =1-\tilde{\gamma}_{11} \bar{\Delta}_{1}\left(j \omega_{0}\right)-\tilde{\gamma}_{22} \bar{\Delta}_{2}\left(j \omega_{0}\right) \\
& =1-\tilde{\gamma}_{11} \operatorname{Re} \bar{\Delta}_{1}\left(j \omega_{0}\right)-\tilde{\gamma}_{22} \operatorname{Re} \bar{\Delta}_{2}\left(j \omega_{0}\right)
\end{aligned}
$$


To see the last equality, we note that

$$
\operatorname{Im} \bar{\Delta}_{k}\left(j \omega_{0}\right)=\frac{1}{\left|1-\beta \Delta_{k}\left(j \omega_{0}\right)\right|^{2}} \operatorname{Im} \Delta_{k}\left(j \omega_{0}\right),
$$

for $k=1,2$. Thus, $\tilde{\gamma}_{11} \operatorname{Im} \bar{\Delta}_{1}\left(j \omega_{0}\right)+\tilde{\gamma}_{22} \operatorname{Im} \bar{\Delta}_{2}\left(j \omega_{0}\right)$ is equal to

$$
\frac{\mu(\alpha-\beta)\left(z \operatorname{Im} \Delta_{1}\left(j \omega_{0}\right)+(1-z) \operatorname{Im} \Delta_{2}(j \omega)\right)}{z\left|1-\beta \Delta_{1}\left(j \omega_{0}\right)\right|^{2}+(1-z)\left|1-\beta \Delta_{2}\left(j \omega_{0}\right)\right|^{2}},
$$

which is equal to 0 by the first condition in (16). Now, notice the following relationship between $\operatorname{Re} \bar{\Delta}_{k}\left(j \omega_{0}\right)$ and $\operatorname{Sect}\left(\Delta_{k}\left(j \omega_{0}\right)\right)$

$$
1+(\beta-\alpha) \operatorname{Re} \bar{\Delta}_{k}\left(j \omega_{0}\right)=\frac{\operatorname{Sect}\left(\Delta_{k}\left(j \omega_{0}\right)\right)}{\left|1-\beta \Delta_{k}\left(j \omega_{0}\right)\right|^{2}},
$$

for $k=1,2$. This gives the following equalities

$$
\begin{aligned}
& \operatorname{det}\left(I-\tilde{\Gamma} \Delta\left(j \omega_{0}\right)\left(I-\beta \Delta\left(j \omega_{0}\right)\right)^{-1}\right) \\
& =1-\tilde{\gamma}_{11} \operatorname{Re} \bar{\Delta}_{1}\left(j \omega_{0}\right)-\tilde{\gamma}_{22} \operatorname{Re} \bar{\Delta}_{2}\left(j \omega_{0}\right) \\
& =\mu \frac{z \operatorname{Sect}\left(\Delta_{1}\left(j \omega_{0}\right)\right)+(1-z) \operatorname{Sect}\left(\Delta_{2}\left(j \omega_{0}\right)\right)}{z\left|1-\beta \Delta_{1}\left(j \omega_{0}\right)\right|^{2}+(1-z)\left|1-\beta \Delta_{2}\left(j \omega_{0}\right)\right|^{2}}+1-\mu
\end{aligned}
$$

Therefore, by the second condition in (16), we conclude that the determinant of $\left(I-\tilde{\Gamma} \Delta\left(j \omega_{0}\right)\left(I-\beta \Delta\left(j \omega_{0}\right)\right)^{-1}\right)$ is equal to 1 when $\mu=0$ and is less than or equal to 0 when $\mu=1$. Hence, the determinant, as a function of $\mu$, has a zero crossing in the interval $(0,1]$, which in turn implies that $\operatorname{det}\left(I-\Gamma \Delta\left(j \omega_{0}\right)\right)$ must take 0 value for a $\mu \in(0,1]$. Since the network is at best neutrally stable at the zero crossing, we have constructed a connection matrix $\Gamma$ which leads to a network that is not strictly stable. This concludes the proof. 